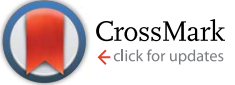

Cite this: RSC Adv., 2016, 6, 47164

\title{
Purification method dependent fluorescence from nitrogen-vacancy (NV) centers of nano-diamonds $\uparrow$
}

\author{
Ravi Kumar, ${ }^{\text {a }}$ S. J. Yoon, ${ }^{\text {}}$ K. G. Lee, ${ }^{\text {b }}$ Prabir Pal, ${ }^{a}$ R. P. Pant, ${ }^{a}$ C. K. Suman, ${ }^{a}$ \\ S. R. Dhakate, ${ }^{a}$ Raj Kumar, ${ }^{c}$ Devesh K. Avasthicd ${ }^{c d}$ and Dilip K. Singh ${ }^{\star a}$
}

Fluorescent nanodiamonds (FNDs) with high photo stability at a subwavelength scale are highly desirable for nano-photonics and bio-imaging applications. Nanodiamonds (NDs) with embedded fluorescent color centers made by ion-implantation need to be purified to remove the $\mathrm{sp}^{2}$ layer on their surfaces which significantly degrades the optical properties. In this work, we discuss the structural and photo physical properties of NDs containing nitrogen-vacancy (NV) centers prepared by two different purification methods; chemical etching $\left(\mathrm{H}_{2} \mathrm{SO}_{4}: \mathrm{HNO}_{3}\right)$ and air oxidation $\left(450{ }^{\circ} \mathrm{C}\right)$. Chemically etched NDs show better uniformity in their shape, de-aggregation and higher dispersibility in water as compared to air oxidized ones. On the other hand it is observed that air oxidation is more effective in removing the $\mathrm{sp}^{2}$ layer and allows a higher fluorescence photon flux. Therefore, we suggest that air oxidation is more appropriate for bright fluorescent sources, and chemical etching is more appropriate for fluorescent markers in bio-imaging applications with high uniformity in shape and good dispersibility.

Received 18th January 2016 Accepted 29th April 2016

DOI: $10.1039 / \mathrm{c} 6 \mathrm{ra01510 \textrm {g }}$

www.rsc.org/advances are the point defect centres consisting of a nitrogen atom at regular substitutional site and an adjacent lattice vacancy in regular diamond matrix. NV centres exhibits strong absorption near $532 \mathrm{~nm}$ and broad emission peak at $\sim 700 \mathrm{~nm}$. NV ${ }^{-}$centres have high quantum yield $(\phi \sim 1),{ }^{20}$ high absorption cross section $\left(5 \times 10^{-17} \mathrm{~cm}^{2}\right)$ and no photo-bleaching. ${ }^{21,22}$ Due to high Debye temperature of diamond and large energy band gap (5.5 $\mathrm{eV}$; ; phonon interactions with $\mathrm{NV}$ centres are negligible. ${ }^{23}$ Hence; NV centres behave as single atom like emission centres and shows this quantum mechanical behaviour up to the room temperature. FNDs show fluorescence resonance energy transfer (FRET) efficiency of about $\sim 30 \%{ }^{24}$ FNDs of size $4-100 \mathrm{~nm}$ has potential to be utilized as platform to incorporate multiple functionalities into single particle including payload of imaging probes or, anticancer drug molecule or, both. But, in the infrared region, the NV centre has 5 times lower brightness than usual fluorophores such as IRDye-800CW, which can be compensated by creating multiple color centers.

NDs have been synthesized by several methods: high pressure high temperature (HPHT) growth, detonation of hydrocarbons, pulsed laser deposition and using different CVD techniques. ${ }^{25-29}$ Large abundance of nitrogen and lower energy requirements leads to their addition in the diamond structure during growth. ${ }^{25}$ Entropy dictated disorderness allows a small concentration of substitutional vacant sites during growth. ${ }^{30}$ These vacancies migrate at high temperature and are trapped by substitutional nitrogen atoms to form NV center. It has been intensively explored to experimentally increase the density of the vacancy and the resulting NV centers.

\author{
nplindia.org \\ ${ }^{b}$ Department of Physics, Hanyang University, Seoul-133-791, Republic of Korea \\ 'Inter University Accelerator Centre, Aruna Asaf Ali Marg, New Delhi-110067, India \\ ${ }^{d}$ Amity Institute of Nanotechnology, Amity University, Noida-201313, India \\ $\dagger$ Electronic supplementary information (ESI) available. See DOI: \\ 10.1039/c6ra01510g
}


Non-diamond carbon phases present at the surface of NDs are detrimental to the fluorescence efficiency. It is important to remove non-diamond layers to obtain high quality FNDs. Previous reports show that different purification method adopted to oxidize the outer surface for purification of nondiamond carbon impurities forms different oxygen and nitrogen related functional groups to variety of extent. ${ }^{26,31,32}$ The effect of different functional groups on the fluorescence of NDs is still an open question. ${ }^{33}$ In this work, we study the structural, chemical, and photo physical properties of NV centers in NDs prepared by two purification methods: chemical etching and air oxidation. Such information is highly required to check the suitability of NV centers embedding NDs for bio-imaging and other nano photonic applications.

\section{Experimental details}

\section{Raw materials, chemicals and milling materials and methods}

Commercially procured HPHT grown micro-diamonds (element six, MICRON + MDA M0.10) were milled using high energy planetary ball mill (Insmart system, model no. PBM07) using steel balls of $10 \mathrm{~mm}$ diameter at $250 \mathrm{rpm}$. All the following milling processes were carried out in the intervals of one hour with 30 minutes break in between. The procured initial sample is referred as ND0. Initially, ND0 was dry milled for 9 hours (referred as ND9) and subsequently wet milled with water for 11 hours (referred as ND20). Further, resulting slurry was milled with $\mathrm{NaCl}$ ( $1: 7$ weight ratio) for additional 15 hours (referred as ND35). $\mathrm{NaCl}$ crystals assist in efficient mechanical energy transfer to the diamond particles. Post milling $\mathrm{NaCl}$ was removed from ND35 slurry by repeated rinsing with water and centrifugation. The graphitic and amorphous carbon present in the sample was purified by two different oxidation methods: (I) through acid reflux and (II) by air oxidation.

\section{Method-I: purification by acid reflux}

In method-I, ND35 slurry was refluxed with conc. $\mathrm{H}_{2} \mathrm{SO}_{4}$ and conc. $\mathrm{HNO}_{3}(3: 1 \mathrm{v} / \mathrm{v}$ ratio $)$ at $80^{\circ} \mathrm{C}$ for 8 hours. Subsequently, it was five times rinsed with double distilled water and centrifuged. Obtained sample was dried in oven for 24 hours at $60^{\circ} \mathrm{C}$. Sample appeared brownish due to iron contamination from the steel vessel and the balls used for milling. Dried sample were crushed with mortar-pestle and were treated with conc. $\mathrm{HCl}$ for 4 hours to remove iron particles. $\mathrm{HCl}$ treatment leads effective etching of iron and results into de-aggregation of NDs by breakage of inter-particle covalent bonding by Fe(II) ions (C-FeC). $\mathrm{HCl}$ treated sample was diluted with water and centrifuged five times to remove acid content. After each dilution and centrifugation, the one third of solution was kept. Finally, it was oven dried. As purified sample has been referred as "ND35R8HCL". The yield of method-I was $57.1 \mathrm{wt} \%$.

\section{Method-II: purification by air oxidation}

In method-II, ND-35 slurry was oxidized under ambient air flow at $450{ }^{\circ} \mathrm{C}$ using rapid thermal annealing at $\left(50{ }^{\circ} \mathrm{C} \mathrm{min}^{-1}\right)$ for 6 hours to remove inter particle graphitic and amorphous carbon layers. Air oxidation etches the non-diamond carbon from the inter-particle sites and outer surface of agglomerates with the addition of oxygen containing functional groups. On air oxidation, iron gets exposed on the outer surface, resulting into apparent reddish color. To remove iron impurities, it was treated with $\mathrm{HCl}$ for 4 hours and 5 times washed with water, centrifuged and was oven dried at $60^{\circ} \mathrm{C}$. This sample is referred as "ND35-OXDHCL". Method-II gives yield of $58.9 \mathrm{wt} \%$.

\section{NV-center creation by ion-irradiation}

Initial sample (ND0) and purified samples (ND35-R8HCL and ND35-OXDHCL) were ion-irradiated with $\mathrm{He}^{+}$ion beams for engineering of $\mathrm{NV}$ color centers at Inter-University Accelerator Center, New Delhi using custom made IUAC $50 \mathrm{keV}$ accelerator (energy $35 \mathrm{keV}$, fluence $2 \times 10^{13}$ ions per $\mathrm{cm}^{2}$, vacuum $10^{-6}$ Torr). Irradiated samples were annealed at $700{ }^{\circ} \mathrm{C}$ for 2 hours at rotary vacuum level.

\section{Characterization}

The particle size and shape of the samples were characterized using scanning electron microscope (SEM) (model no. VP-EVO, MA-10, Carl-Zeiss, UK), transmission electron microscope (TEM) (model no. FEI, Tecnai T30). Raman and fluorescence spectra of the samples were recorded using $514 \mathrm{~nm}$ excitation from $\mathrm{Ar}^{+}$ion-laser and single monochromator equipped microRaman spectrometer with $50 \times$ objective lens using commercial system (Renishaw in Via Raman Microscope) in the backscattering geometry. Particle size analysis was carried out using blue wave laser diffraction analyzer based on Mie scattering (model no. Bluewave particle analyzer). X-ray diffraction pattern (XRD) was recorded using Rigaku made powder X-ray diffractometer (model-XRG $2 \mathrm{~kW}$ ) at a scanning rate of 0.02 degree per $\mathrm{s}$ in the $2 \theta$ range from $20^{\circ}$ to $70^{\circ}$ operating at $40 \mathrm{kV}, 30 \mathrm{~mA}$ with automatic divergence slit using $\mathrm{Cu}-\mathrm{K}_{\alpha}$ radiation $(\lambda=1.54059 \AA)$. Thermo-gravimetric measurements were carried out at a heating rate of $5{ }^{\circ} \mathrm{C} \mathrm{min}{ }^{-1}$ with $40 \mathrm{sccm} \mathrm{min}^{-1}$ of air flow (using Mettler-Toledo TGA star system). The X-ray photo-electron spectroscopy (XPS) measurements were performed by using an Omicron $\mu$-metal ultrahigh vacuum (UHV) system equipped with a monochromatic Al $\mathrm{K}_{\alpha}$ X-ray source $(h \nu=1486.6 \mathrm{eV})$ and a multi-channeltron hemispherical electron energy analyzer (EA 125). The samples were mounted on the sample plates using spot welded gold foils. An electrical contact was established between the sample surface and Omicron sample plate with a highly conducting UHV compatible silver (Ag) paint in order to avoid charging during the photoemission measurements due to their insulating nature of the samples. The samples were heated in situ at $300{ }^{\circ} \mathrm{C}$ under ultra-high vacuum conditions and then cooled down to room temperature before photoemission measurements. This heating procedure helped us to clean the sample surface as confirmed by the absence of surface adsorbed feature around $\sim 532 \mathrm{eV}$ in the $\mathrm{O} 1 \mathrm{~s}$ core level spectra. The photoemission measurements were performed inside the analysis chamber under a base vacuum of $\sim 5.0 \times 10^{-11} \mathrm{mbar}$ at room temperature. The binding energy of all the spectra was calibrated by considering $\mathrm{Au} 4 \mathrm{f}_{7 / 2}$ at $84.0 \mathrm{eV}$ and the Fermi 
energy $\left(E_{\mathrm{F}}\right)$ from Au foil in electrical contact with the sample. The energy resolution was $400 \mathrm{meV}$ in case of XPS spectra using monochromatic $\mathrm{Al} \mathrm{K}_{\alpha}$ source. We have subtracted the background intensity due to secondary electrons from all the core level spectra using the Shirley and Tougaard background corrections.

\section{Results and discussion}

Scanning electron microscope (SEM) images of initial sample (ND0), 35 hours milled sample (ND35) and purified samples ND35-R8HCL (through chemical etching, method-I) and ND35OXDHCL (through air oxidation, method-II) are shown in Fig. S1 (ESI $\dagger$ ). The average particle sizes were estimated using Image s software over more than 40 particles seen in the micrograph and are enlisted with standard deviation in Table $1 .^{34}$ ND0 shows the average particle size of $127.9 \pm 67.9$ $\mathrm{nm}$, Fig. S1(a). $\dagger$ ND35 shows average particle size of $100.4 \mathrm{~nm}$ with calculated standard deviation of $27.0 \mathrm{~nm}$, Fig. S1(b). $\dagger$ ND35-R8HCL shows average particle size of $91.7 \pm 31.5 \mathrm{~nm}$ (Fig. S1(c) $\dagger$ ), while ND35-OXDHCL shows average particle size of $128.8 \pm 35.9 \mathrm{~nm}$ (Fig. S1(d) $\dagger$ ). Fig. 1 shows the HRTEM micrographs of (a) ND0, (b) ND35, (c) ND35-R8HCl and (d) ND35OXDHCL. The inset of the figures shows selected area electron diffraction (SAED) pattern taken on individual particle. For the analysis of the images and diffraction pattern ImageJ software was used. Fig. 1(a) shows initial particles were obloidal in nature (length $134.5 \mathrm{~nm}$, width $65.5 \mathrm{~nm}$ ). Milling for 35 hours (ND35) results into shape change from obloidal to mixture of rectangles and triangles (Fig. 1(b)). Milled sample also contained particles of very small size $(\sim 10 \mathrm{~nm})$. The average length of the rectangle observed was $36.1 \mathrm{~nm}$ and width of $25.8 \mathrm{~nm}$. ND35-R8HCL shows rectangular particles (length $70.5 \mathrm{~nm}$, width $40.8 \mathrm{~nm}$ ) along with very few triangular structures, Fig. 1(c). While ND35-OXDHCL shows rectangular particles (length $104.1 \mathrm{~nm}$, width $62.6 \mathrm{~nm}$ ) along with a significant numbers of irregular shaped particles Fig. 1(d). ND35-OXDHCL shows relatively higher agglomeration than the ND35-R8HCL. SAED pattern shows narrow ring like structure along with presence of sharp diffraction spots sitting over the rings. The estimated $d$-spacing along with corresponding lattice planes are indicated in insets. ND0 shows sharp spots sitting over a broad diffuse ring, indicating crystalline phase with a small fraction of amorphous carbon present as impurity. ND-35 shows multiple sharp rings with sharp spots sitting over them. Multiple ring structure originates from the impurities impregnated during milling as indicated by additional diffraction rings corresponding to $\mathrm{Fe}$ and $\mathrm{NaCl}$. The initial sample shows diffraction rings with $d$-spacing $0.113 \mathrm{~nm}(220), 0.063 \mathrm{~nm}(400)$ and 0.052 $\mathrm{nm}$ (unidentified plane) of diamond. The assignment of lattice plane to the experimentally observed $d$-spacing has been done with reference to PCPDF data base \# 060675. As per the PCPDF data base \# 060675, the diamond shows diffraction spots corresponding to $d$-spacing of $0.206 \mathrm{~nm}$ (111), $0.126 \mathrm{~nm}$ (220), $0.107 \mathrm{~nm}$ (311), $0.089 \mathrm{~nm}$ (400), $0.082 \mathrm{~nm}$ (unassigned) respectively. The experimentally observed $d$-spacing from SAED pattern shows lower $d$-spacing than as expected from standard PCPDF database. This indicates initial nanoparticles ND0 contain compressive lattice strain. Compressive strain relaxes after milling as indicated by change in the $d$-spacing from $0.113 \mathrm{~nm}$ to $0.136 \mathrm{~nm}$ for (220) plane, $0.063 \mathrm{~nm}$ to 0.066 $\mathrm{nm}$ for (400) plane. The SAED pattern of purified samples shows that acid refluxed NDs contains higher strain than the sample purified through air oxidation. The observed difference in the shape of the NDs purified through two methods in TEM arises due to difference in the amount of strain involved.

Fig. 2 shows the powder X-ray diffraction (XRD) pattern of sample milled for different periods and after purification. ND0 shows diffraction peak at $\sim 43.8^{\circ}(111)$ and $75.2^{\circ}(220)$. Experimentally observed diffraction peaks are fitted with Voigt line shape. The change in the line profile parameters along with estimated crystallite size using Debye-Scherrer equation are summarized in Table S1 (ESI $\dagger$ ). Fitting of (111) peak requires a weaker shoulder on lower Bragg angle $43.703^{\circ}$ (crystallite size $\sim 16.3 \mathrm{~nm}$ ) in addition to $43.888^{\circ}$ peak (crystallite size $\sim 25.5$ $\mathrm{nm})$. With increasing milling period, the oscillating shift in (111) peak position indicates periodic creation and relaxation of compressive strain respectively from mechanical impacts. ${ }^{35}$ Variation in the strain leads to varying disorders in the particles as reflected in terms of width (FWHM) change with increasing milling hours. No significant change in the crystallite size was observed upon dry milling for 09 hours. Additional wet milling for 11 hours shows reduction in the crystalline disorderness in terms of significant decrease in FWHM of (111) peak, but not any significant change in crystallite size. XRD measurements reveals that even prolonged milling, with different milling

Table 1 Particle size analysis

\begin{tabular}{|c|c|c|c|c|c|}
\hline \multirow[b]{3}{*}{ Sample } & \multicolumn{4}{|c|}{ Particle size (nm) } & \multirow[t]{2}{*}{$\begin{array}{l}\text { Crystallite } \\
\text { size }(\mathrm{nm})\end{array}$} \\
\hline & \multicolumn{2}{|l|}{ HRTEM } & \multirow[b]{2}{*}{ SEM } & \multirow[b]{2}{*}{ DLS } & \\
\hline & Length & Width & & & XRD \\
\hline ND0 & 134.5 & 65.5 & $127.9 \pm 67.9$ & 160.0 & 16.3 and 25.6 \\
\hline ND35 & 36.1 & 25.8 & $100.4 \pm 27.0$ & $80.0,200.0,350.0$ & 23.2 \\
\hline ND35-R8HCL & 70.5 & 40.8 & $91.7 \pm 31.5$ & 120.0 & 18.7 and 25.8 \\
\hline ND35-OXDHCL & 104.1 & 62.6 & $128.8 \pm 35.9$ & 120.0 & 18.8 and 27.4 \\
\hline
\end{tabular}



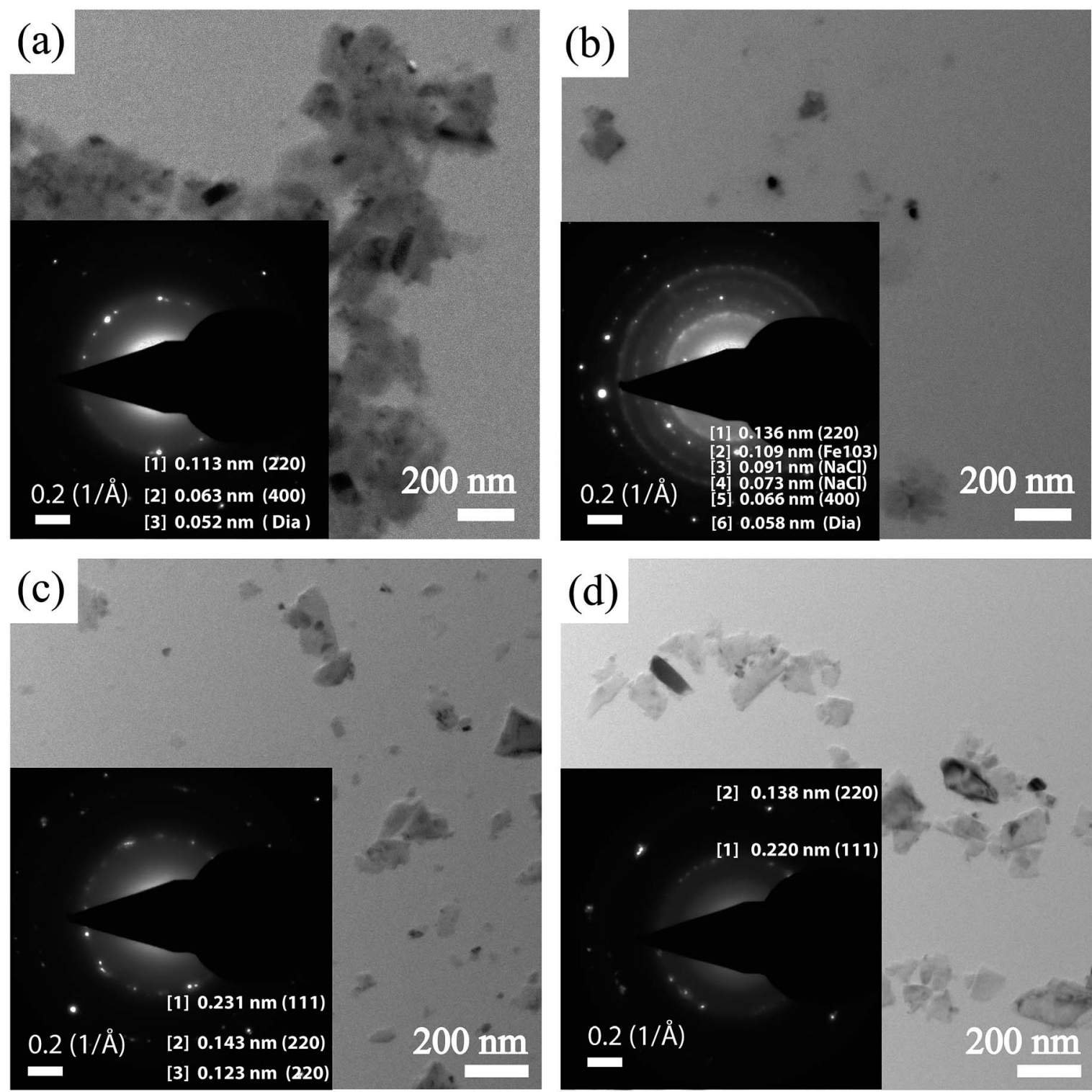

Fig. 1 Shows the TEM (SAED pattern in inset) of (a) initial sample, (b) milled sample ND35, (c) acid refluxed NDs: ND35-R8HCL and (d) air oxidized NDs: ND35-OXDHCL. Corresponding to the observed $d$-spacing in the SAED pattern, lattice planes are marked.

media ( $\mathrm{NaCl} /$ water), the final change in the crystallite size remains minimal. Although from DLS measurements, we observe that the particle size decreases significantly within only 09 hours of milling, Fig. S2 (ESI $\dagger$ ). This indicates that the primary particle size can be reduced only up to prevailing crystallite size and not below it. To reduce the particle size further, mechanical energy must be supplied at nanoscale with zirconium milling (bead assisted sonication disintegration), but large amorphitization of diamond phase and highly chemical resistant nature of zirconia puts the restriction on this method. ${ }^{36}$ ND35R8HCL shows crystallite size of $25.8 \mathrm{~nm}$ along with a smaller fraction of particles with crystallite size $18.7 \mathrm{~nm}$. While ND35OXDHCL shows crystallite size of range $27.4 \mathrm{~nm}$ along with a fraction of particles of crystallite size $18.8 \mathrm{~nm}$.

Fig. 3(a) shows Raman spectra of ND0, ND9, ND20 and ND35 (initial, 09 hours, 20 hours and 35 hours milled) samples. These spectra were fitted with Lorentzian line shapes with slanted linear background to monitor the change in spectral parameter with milling time and on purification as shown in Fig. 3(b) for ND0. Changes in the spectral parameters are summarized in Table SII. $\dagger$ Raman spectra show two prominent features in the range $1300-1400 \mathrm{~cm}^{-1}$ and $1500-$ $1600 \mathrm{~cm}^{-1}$ known as D and $\mathrm{G}$ modes of carbon. D-mode of the sample consists of two Lorentzian components namely 1331.8 and $1351.5 \mathrm{~cm}^{-1}$ with FWHM of 5.9 and 62.3 respectively. Peak at $1331.8 \mathrm{~cm}^{-1}$ is assigned to triply degenerate $\left(F_{2 g}\right)$ zone center phonon mode of diamond lattice (arising from $O_{\mathrm{h}}^{7}$ space group), ${ }^{37}$ while peak at $1351.5 \mathrm{~cm}^{-1}$ with relatively larger peak width is assigned to dis-ordered $\mathrm{sp}^{3}$ bonded carbon structure/ amorphous carbon. ${ }^{38}$ Peak at $1351.5 \mathrm{~cm}^{-1}$ arises from lattice distortion induced breakdown of momentum conservation requirement, which activates phonons at the interior $K$ point 
(a)
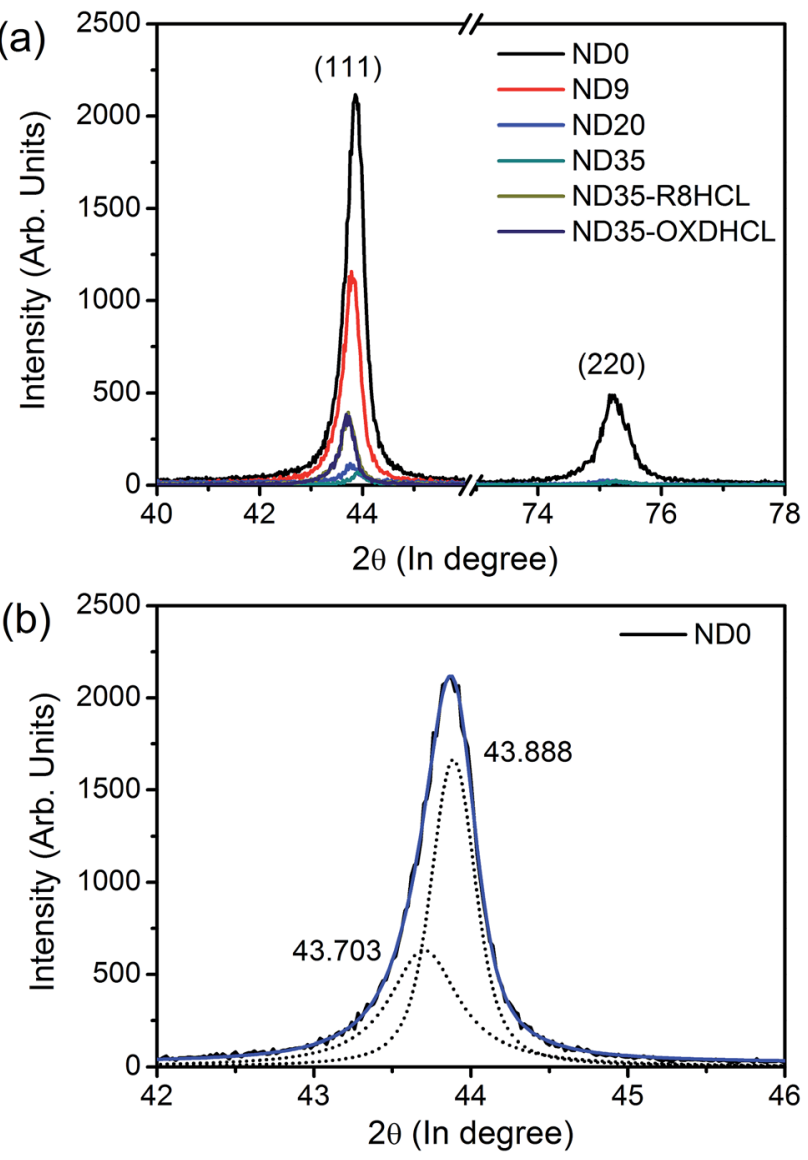

Fig. 2 (a) Shows XRD pattern of the diamond particles milled for 0 hours (ND0), 9 hours (ND9), 20 hours (ND20) and 35 hours (ND35) and purified NDs ND35-R8HCL and ND35-OXDHCL. (b) Fitted (111) peak of NDO using two Voigt components.

of the Brillouin zone $(q \neq 0) \cdot{ }^{39}$ In addition to D-modes, spectra show peaks at $1482.3 \mathrm{~cm}^{-1}, 1578.7 \mathrm{~cm}^{-1}, 1615.7 \mathrm{~cm}^{-1}$ and $1688.2 \mathrm{~cm}^{-1}$. Peak at $\sim 1578.8 \mathrm{~cm}^{-1}$ is assigned to the graphitic G-mode of carbon arising from intra layer doubly degenerate $\mathrm{E}_{2 \mathrm{~g}}$ mode of $\mathrm{sp}^{2}$ bonded graphitic carbon structure, while peak at $1615.7 \mathrm{~cm}^{-1}$ is assigned to defect related $\mathrm{D}^{\prime}$-mode of carbon. ${ }^{40} \mathrm{D}^{\prime}$-band arises due to breakdown of $q=0$ wave vector selection rule and has been observed for graphite samples with crystallite sizes less than $10 \mathrm{~nm} .{ }^{41}$ In few samples, we observed peaks at $\sim 1480 \mathrm{~cm}^{-1}$ i.e. in between D and $\mathrm{G}$ modes. Origin of this peak has been assigned to polyacetylene and has nothing to do with $\mathrm{C}-\mathrm{C} \mathrm{sp}{ }^{3}$ carbon. ${ }^{42,43}$ The peak position of $F_{2 g}$ mode changes from $1331.8 \mathrm{~cm}^{-1}$ to $1330.8 \mathrm{~cm}^{-1}, 1332.5$ $\mathrm{cm}^{-1}, 1331.5 \mathrm{~cm}^{-1}$ after 9 hours, 20 hours and 35 hours of milling. The back and forth oscillating Raman peak position of $\mathrm{F}_{2 \mathrm{~g}}$ mode arises from the compressive and elongation strain produced upon milling. ${ }^{44}$ The observed fluctuations in the peak position about $1331.8 \mathrm{~cm}^{-1}$ is in agreement with XRD measurements which show oscillatory shifts in the $2 \theta$ position with milling time. Analogous to the D-band, G-band also shows variation in the peak position with milling time. Peak observed at $1578.7 \mathrm{~cm}^{-1}$ shifts to $1583.4 \mathrm{~cm}^{-1}, 1584.4 \mathrm{~cm}^{-1}$ and $1586.8 \mathrm{~cm}^{-1}$ upon 09 hours, 20 hours and 35 hours of milling. Further the FWHM of G peak monotonically decreases with increasing milling time indicating increasing graphitic ordering with milling impacts on diamond particles. It is in agreement with the particle size measurements which show the agglomeration of the particles.

Fig. 3(c) shows the Raman spectra of as procured, milled and purified samples from the two different methods. The estimated relative content of the $\mathrm{sp}^{2}$ and $\mathrm{sp}^{3}$ hybridized carbon content is estimated from the area under D and G modes in the Raman spectra. Initial sample ND0 contains $66.4 \mathrm{wt} \%$ of $\mathrm{sp}^{2}$ carbon, $12.4 \mathrm{wt} \%$ of $\mathrm{sp}^{3}$ diamond structure and $21.2 \mathrm{wt} \%$ of disordered $\mathrm{sp}^{3}$ carbon. Post milling the sample purified through acid reflux contains $27.3 \mathrm{wt} \%$ of diamond $\mathrm{sp}^{3}$ phase, $30.6 \mathrm{wt} \%$ of disordered $\mathrm{sp}^{3}$ structure and $42.1 \mathrm{wt} \%$ of $\mathrm{sp}^{2}$ carbon. Air oxidized sample contains $57.4 \mathrm{wt} \%$ of $\mathrm{sp}^{3}$ hybridized carbon, $23.2 \mathrm{wt} \%$ of disordered carbon and just $19.4 \mathrm{wt} \%$ of $\mathrm{sp}^{2}$ carbon. Purification by air oxidation appears to be better method for removal of $\mathrm{sp}^{2}$ carbon impurities from Raman spectroscopy measurements. In addition to it, amount of disordered carbon structure is also relatively less in case of purification by air oxidation. Fig. 3(d) shows the comparative Raman spectra of initial and purified samples before irradiation (ND0, ND35-R8HCL and ND35-OXDHCL) and after irradiation ND0-IRR, ND35-R8HCLIRR and ND35-OXDHCLIRR. ND0-IRR shows decrease in the $\mathrm{sp}^{3} \mathrm{wt} \%$ content from $12.4 \mathrm{wt} \%$ to 0.4 wt $\%$ of diamond content. The acid refluxed and ion-irradiated sample (ND35-R8HCLIRR) shows decrease in the diamond content from $27.4 \mathrm{wt} \%$ to $0.5 \mathrm{wt} \%$, while air oxidized and ionirradiated sample (ND35-OXDHCLIRR) shows decrease in the diamond content from $57.4 \mathrm{wt} \%$ to $12.5 \mathrm{wt} \%$. Ion-irradiation leads to significant amount of $\mathrm{sp}^{3}$ to $\mathrm{sp}^{2}$ conversion. Additionally, the amount of disordered $\mathrm{sp}^{3}$ carbon content $(30.6 \mathrm{wt} \%)$ is higher in sample purified through acid reflux as compared to air oxidized sample (23.2 wt\%). Interestingly, the sample purified through air oxidation is more resistant to $\mathrm{He}^{+}$-ion irradiation for creating NV centers.

Fig. 4 shows the X-ray photoelectron spectra (XPS) of ND0, ND35, ND35-R8HCL and ND35-OXDHCL. Fig. 4(a), the survey spectra of these samples show peaks at binding energies at 285.1, 399.2 and 531.3 eV corresponding to $\mathrm{C} 1 \mathrm{~s}, \mathrm{~N} 1 \mathrm{~s}, \mathrm{O} 1 \mathrm{~s}$ cores respectively. Fig. 4(b) shows C 1s core spectra. Fig. 4(c) shows the typical fitting to the $\mathrm{C}$ 1s core level spectra of the ND0 using XPSPEAK 4.1. Similarly, N 1s and O 1s core level was fitted. The summary of the fitted peak profile for all the samples are summarized in Table-SIII and SIV (ESI $\dagger$ ) for C 1 s core and in Table-SV (ESI $\dagger$ ) for $\mathrm{N} 1 \mathrm{~s}$ and $\mathrm{O}$ 1s cores. Inset of the Fig. 4(c) shows presence of peak at $400.1 \mathrm{eV}$ for initial sample ND0 indicating presence of nitrogen dopants in diamond lattice. Fig. 4(d) shows the $\mathrm{O} 1 \mathrm{~s}$ core spectra. The atomic concentrations of carbon, oxygen and nitrogen were estimated using corresponding peak area after background subtraction normalized to the inelastic mean free path, photoionization cross-section $(\sigma)$ and analyzer transmission function ( $2^{\text {nd }}$ order approximation). The photoionization cross-section $(\sigma)$ was taken as 0.013 for $\mathrm{C}$ 1s core, 0.040 for $\mathrm{O} 1 \mathrm{~s}$ core and 0.024 for $\mathrm{N} 1 \mathrm{~s}$ core. Inelastic mean free path (IMFP) was taken as $33.03 \AA$, 26.53 $\mathrm{A}$ and $29.51 \AA$ for $\mathrm{C}$ 1s core, $\mathrm{O}$ 1s core and $\mathrm{N}$ 1s core respectively. The 

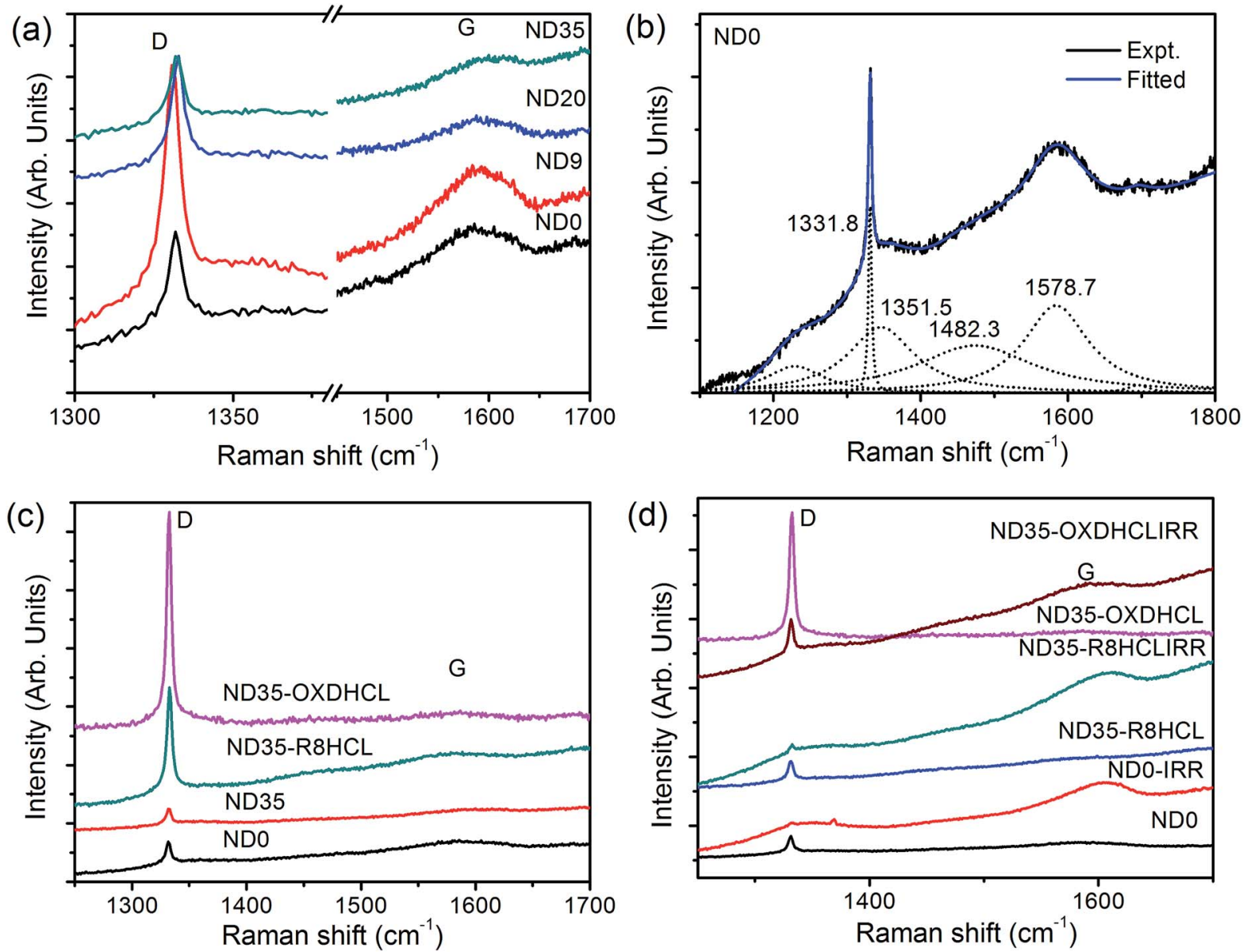

Fig. 3 (a) Raman spectra of diamond milled for different duration. (b) Fitted spectral line profile of ND0 using Lorentzian line shapes with slanted linear background. (c) Raman spectra of ND0, ND35, ND35-R8HCL and ND35-OXDHCL. (d) Raman spectra of ND0, ND35, ND35-R8HCL and ND35-OXDHCL before and after irradiation. Sharp D-band component (FWHM $6 \mathrm{~cm}^{-1}$ ) represents diamond content, broad D-band represents disordered $\mathrm{sp}^{3}$ carbon and $\mathrm{G}$-band represents graphitic carbon.

compositions of different samples obtained from this procedure are listed in Table-SVI (ESI $\dagger$ ).

ND0 shows peaks of nitrogen and oxygen in addition to carbon core, Fig. 4(a). C 1s core spectra of ND0 exhibits peak at $286.2 \mathrm{eV}$ and $286.9 \mathrm{eV}$, corresponding to $\mathrm{sp}^{2}$ and $\mathrm{sp}^{3}$ hybridized carbon. These peaks are shifted towards high binding energy as compared to expected $284.3 \mathrm{eV}$ and $285.2 \mathrm{eV}$ peak from $\mathrm{sp}^{2}$ and $\mathrm{sp}^{3}$ carbon respectively. ${ }^{45,46}$ The initial sample is estimated to contain $79.9 \mathrm{wt} \%$ of $\mathrm{sp}^{2}$ carbon and $20.0 \%$ of $\mathrm{sp}^{3}$ carbon. This is in close agreement with estimated $\mathrm{sp}^{2}(66.4 \%), \mathrm{sp}^{3}$ carbon $(12.4 \%)$ and disordered $\mathrm{sp}^{3}$ carbon $(21.1 \%)$ from Raman spectroscopy. Since X-rays have finite penetration depth $(\sim 4 \mathrm{~nm})$, XPS studies are more sensitive to surface stoichiometry and it shows that the outer surface of the particles contains higher graphitic carbon. The $\mathrm{sp}^{2}$ hybridized carbon and outer surface atoms terminated with oxygen containing functional groups (as evidenced from observation of $\mathrm{O} 1 \mathrm{~s}$ core) stabilizes the nanoparticles by terminating dangling bonds. Two plasmon loss peaks are also observed at $313.0 \mathrm{eV}$ and $321.0 \mathrm{eV}$ with an unidentified peak at $340.1 \mathrm{eV}$. Shift in the $\mathrm{sp}^{2}$ and $\mathrm{sp}^{3}$ bonded carbon peaks against the reference binding energy for these two cores is mainly due to defects and small primary particle size. Defects are present at the surface due to the abrupt discontinuity of the diamond phase and leads to the disturbance of charge neutrality. The band bending increases the binding energy of $\mathrm{C} 1 \mathrm{~s}$ spectra. ${ }^{47}$ In addition to band bending, it may have contribution from charging effects due to insulating diamond nanoparticles. ${ }^{48}$ ND35 shows peaks at slightly lower binding energy at $285.2 \mathrm{eV}$ and $285.7 \mathrm{eV}$ for $\mathrm{sp}^{2}$ and $\mathrm{sp}^{3}$ hybridized carbon amounting to $80.2 \mathrm{wt} \%$ and $19.8 \mathrm{wt} \%$ respectively. In case of ND35, plasmon loss peaks are also observed at smaller binding energies (309.0 eV and $319.5 \mathrm{eV}$ ) supporting the understanding that in case of milled sample presence of metallic impurities reduces the charging effects. Change in the binding energy for the carbon core reveals that iron contamination leads to the agglomeration of primary particles along with abrupt reduction in the surface defects and reduction in the band bending contribution. This is in agreement with agglomeration observed in the particle size analysis (Fig. S2(d); ESI†).

The sample purified through acid reflux ND35-R8HCL shows oxidized surface features with the reduced $\mathrm{sp}^{2}$ carbon near the 

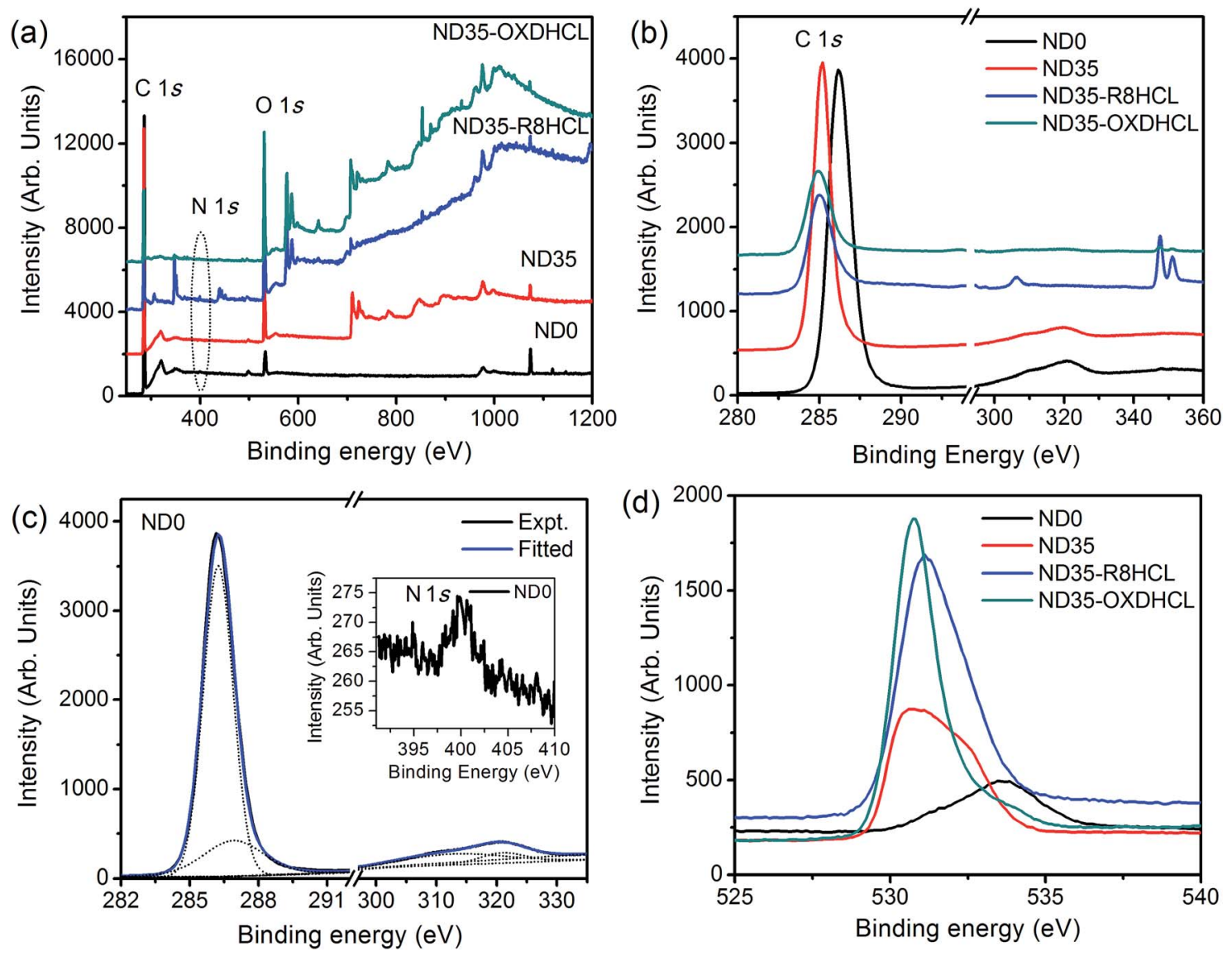

Fig. 4 Shows XPS spectra. (a) Shows the survey scan spectra of ND0, ND35, ND35-R8HCL and ND35-OXDHCL. (b) Shows the C 1s carbon core peak. (c) Fitting of $\mathrm{C} 1 \mathrm{~s}$ core peak, typically shown for ND0. Inset shows peak observed in the range 395-410 eV corresponding to N 1 s core. (d) Shows $\mathrm{O}$ 1s core-spectra.

surface. In case of ND35-R8HCL, the binding energies of $\mathrm{sp}^{2}$ $(285.0 \mathrm{eV})$ and $\mathrm{sp}^{3}(286.2 \mathrm{eV})$ hybridized carbon atoms are lower than the initial ND0. Chemical etching of graphitic and amorphous carbon layers results into observed lower binding energy. ND35-R8HCL contains $26.1 \mathrm{wt} \% \mathrm{sp}^{2}$ carbon and $17.3 \mathrm{wt} \% \mathrm{sp}^{3}$ carbon. Presence of different oxygen containing groups is confirmed by presence of peaks at $288.8 \mathrm{eV}, 294.4 \mathrm{eV}$, and 297.0 $\mathrm{eV}$ (corresponding to $\mathrm{C}-\mathrm{O}, \mathrm{C}=\mathrm{O}$ and $\mathrm{O}=\mathrm{C}-\mathrm{O}$ species). ${ }^{49}$ Functionalized carbon atoms amount to $56.6 \mathrm{wt} \%$ of the sample. Reduction of the graphitic layers is in agreement with observations in Raman spectra where the G band is diminished in the acid refluxed sample. Reduction of the graphitic layers and subsequent de-agglomeration due to the presence of the oxygen containing groups is also confirmed by dynamic light scattering based particle size analysis measurements for acid refluxed sample. Also, a weak plasmon loss peak is observed at $306.3 \mathrm{eV}$. On the other hand, ND35-OXYHCL contains $77.3 \mathrm{wt} \%$ $\mathrm{sp}^{2}$ carbon $(284.9 \mathrm{eV})$ and $14.2 \mathrm{wt} \% \mathrm{sp}^{3}$ carbon $(286.5 \mathrm{eV})$. In addition, it contains two peaks at $291.1 \mathrm{eV}$ and $293.7 \mathrm{eV}$ showing presence of oxygen containing groups amounting to $8.5 \mathrm{wt} \%$ of the sample. Plasmon loss peaks are observed at 307.6 $\mathrm{eV}$ and $318.9 \mathrm{eV}$.

Irradiated sample ND0-IRR contains $64.3 \mathrm{wt} \% \mathrm{sp}^{2}$ and $35.7 \mathrm{wt} \% \mathrm{sp}^{3}$ carbon with peaks at $285.3 \mathrm{eV}$ and $286.5 \mathrm{eV}$ respectively. Irradiation and subsequent annealing result into modification of outer surface and leads to reduction of $\mathrm{sp}^{2}$ carbon on the surface. $\mathrm{sp}^{2}$ and $\mathrm{sp}^{3}$ peaks were found to shift towards lower binding energy as compared to ND0 possibly due to the surface defect reduction on annealing. Irradiation of the acid refluxed sample (ND35-R8HCLIRR) leads to more graphitization on the outer core and the oxygen related functional groups are detached from the outer surface. In case of ND35R8HCLIRR, the $\mathrm{sp}^{2}$ carbon $(285.4 \mathrm{eV})$ is found to account for $72.4 \mathrm{wt} \%, \mathrm{sp}^{3}$ carbon for $23.1 \mathrm{wt} \%$ (285.9 eV peak) and functionalized carbon atoms amounts to $4.4 \mathrm{wt} \%$. Peak at $293.7 \mathrm{eV}$ shows the presence of functional oxygen groups at the outer surface, survived by irradiation and annealing. While, ND35OXDHCLIRR is found to contain 16.7 and $5.6 \mathrm{wt} \%$ of $\mathrm{sp}^{2}$ and $\mathrm{sp}^{3}$ carbon respectively along with $77.6 \mathrm{wt} \%$ fraction of functionalized carbon atoms. In case of ND35-OXDHCLIRR, the $\mathrm{sp}^{2}$ and $\mathrm{sp}^{3}$ peaks are observed towards higher binding energy side at 288.7 and $288.9 \mathrm{eV}$ respectively. Even the plasmon loss peaks are observed towards higher binding energy side at $314.0 \mathrm{eV}$ and $324.8 \mathrm{eV}$.

Analysis of the N 1s core (Table SV; ESI $\dagger$ ) of ND0 shows peak at $400.1 \mathrm{eV}$. It indicates presence of nitrogen. $\mathrm{N} 1 \mathrm{~s}$ core peak was not observed for ND35 within sensitivity limit of XPS, probably due to lattice relaxation and surface reconstruction during 
milling. Structural reconstructions lead to nitrogen migration towards the center of the primary particles. $\mathrm{N} 1 \mathrm{~s}$ core peak was not observed in all other samples, except ND35-OXDHClIRR. The understanding about presence of substitutional nitrogen atoms on the surface within X-ray penetration depth only for ND35-OXDHCLIRR is not clear to us. Fig. 4(d) shows the O 1s core spectra. Calculated peak area of $\mathrm{O} 1 \mathrm{~s}$ core reveals that more oxygen is incorporated on the diamond particles during milling. Further, the amount of oxygen attached to the particle surfaces increases during purification. ND0 shows the presence of carbon oxygen double bond species $(\mathrm{C}=\mathrm{O})(531.4 \mathrm{eV})$, nitrosyl $(533.6 \mathrm{eV})$, and hydro peroxide groups (536.3 eV) (Table SV; ESI $\dagger) .{ }^{50}$ ND35 shows the presence of nitrosyl $(532.2 \mathrm{eV})$, alcoholic $(530.2 \mathrm{eV})$ and carbon oxygen double bond $(\mathrm{C}=\mathrm{O})$ species $(530.8 \mathrm{eV})$. ND35-R8HCL also reveals the presence of carbon oxygen double bond $(\mathrm{C}=\mathrm{O})$ species $(531 \mathrm{eV})$ and nitrosyl $(532.5$ $\mathrm{eV}$ ) with large surface areas, i.e. surface is dominated by the oxygen containing groups. In case of ND35-OXYHCL in addition to carbon oxygen double bond $(\mathrm{C}=\mathrm{O})$ species and nitrosyl groups (533.8 eV); a prominent peak is also appears at $530.7 \mathrm{eV}$. Interestingly, ND35-R8HCL contains more surface functionalized oxygen than the ND35-OXDHCL.
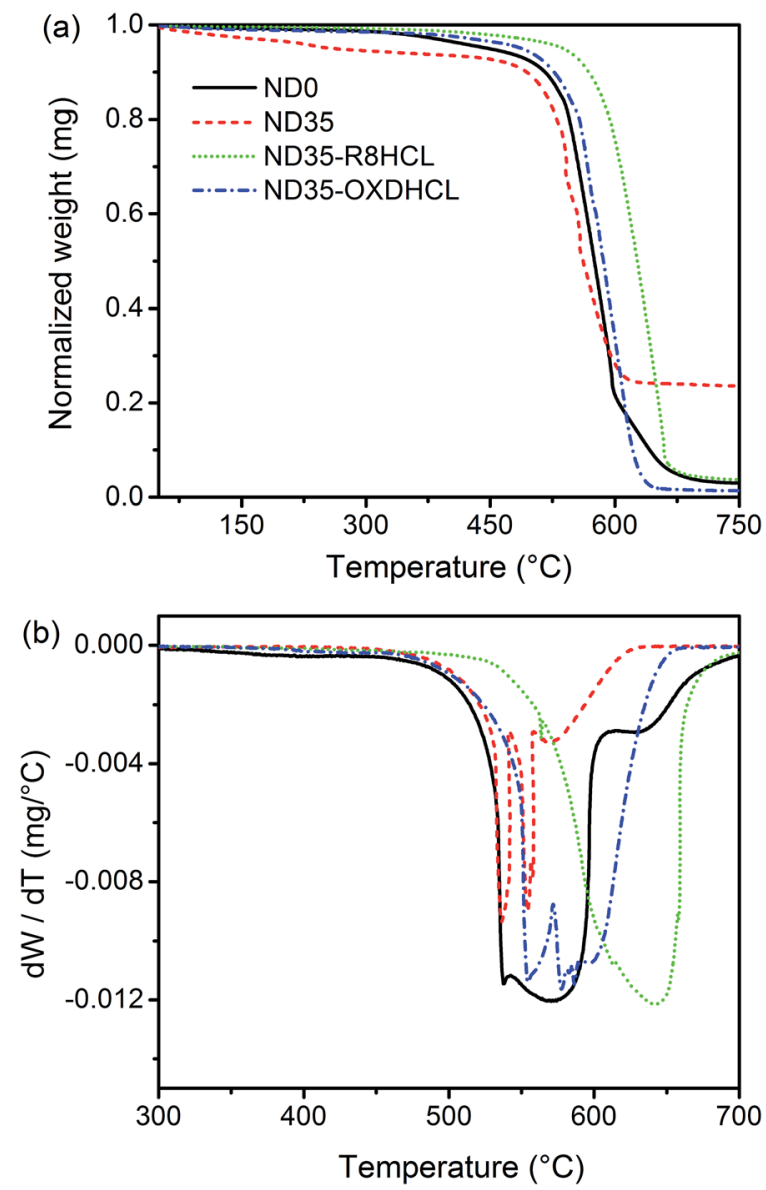

Fig. 5 (a) TGA pattern of ND0, ND35, ND35-R8HCL and ND35OXDHCL. The corresponding DTA pattern is shown in (b). The sample purified through acid reflux shows relatively higher thermal stability than the sample purified through air oxidation.
Fig. 5(a) shows the Thermo-gravimetric analysis (TGA) pattern. ND0 and ND35 shows similar oxidation pattern although the amount of residue left after oxidation differs significantly. The ND0 shows nearly complete oxidation with only $3.1 \mathrm{wt} \%$ as residue, while ND35 shows about $23.8 \%$ residue leftover after oxidation, probably from iron particles incorporated from balls and vials used for milling. ND35-R8HCL shows higher oxidative stability with steeper oxidation. The differential thermo-gravimetric analysis (DTA) curve of ND0 shows three peak structure with corresponding maxima in the oxidation at $397{ }^{\circ} \mathrm{C}, 537{ }^{\circ} \mathrm{C}, 571{ }^{\circ} \mathrm{C}$ and $631^{\circ} \mathrm{C}$ amounting to oxidation of 3.3 $\mathrm{wt} \%, 12.4 \mathrm{wt} \%, 29.8 \mathrm{wt} \%$ and $41.5 \mathrm{wt} \%$ sample respectively indicating presence of four different phases of carbon, Fig. 5(b). The milled sample ND35 also shows four different components at $536{ }^{\circ} \mathrm{C}, 554{ }^{\circ} \mathrm{C}, 558{ }^{\circ} \mathrm{C}$ and $569{ }^{\circ} \mathrm{C}$ accounting for the 22.8 $\mathrm{wt} \%, 16.7 \mathrm{wt} \%, 8.9 \mathrm{wt} \%$ and $6.5 \mathrm{wt} \%$ of the sample respectively. In contrast to the initial and milled samples, the purified sample ND35-R8HCL shows only one maxima in the DTA curve at $642{ }^{\circ} \mathrm{C}$, while in case ND35-OXDHCL three maxima were observed in the DTA curve at $554{ }^{\circ} \mathrm{C}, 577{ }^{\circ} \mathrm{C}$ and $596{ }^{\circ} \mathrm{C}$ representing 18.7, 20.7 and $21.3 \%$ weight loss. In case of sample purified through wet chemical route $3.4 \mathrm{wt} \%$ metallic residue was left, while in case of sample purified through air oxidation $1.3 \mathrm{wt} \%$ of the residue was observed.

Fig. 6(a) shows the fluorescence (FL) spectra. FL spectra were fitted with Gaussian line shape, typically shown for ND35R8HCLIRR in Fig. 6(b). The fitted spectral line profile parameters are listed in Table SVII (ESI $\dagger$ ). ND0 shows very weak and broad emission intensity. Emission intensity of milled sample ND35 is further suppressed extensively due to incorporation of iron particles, a well-known fluorescence quencher. ${ }^{51}$ On purification, ND35-R8HCL and ND35-OXDHCL shows emission intensity similar to ND0. In addition, two weak peaks are seen sitting on the broad background on close observation at 552 and $559 \mathrm{~nm}$. These are D and G Raman modes. Except ND35OXDHCL sample, characteristics emission of NV center is not observed in non-irradiated samples. ND35-OXDHCL shows weak characteristic zero phonon line (ZPL) for $\mathrm{NV}^{\circ}(575 \mathrm{~nm})$ and $\mathrm{NV}^{-}$centers $(638 \mathrm{~nm})$. It is due to relatively efficient iron contamination removal by air oxidation method, which is also confirmed from TGA measurements. The emission intensity was multifold enhanced with creation of NV centers in NDs by ion irradiation. ND0-IRR shows broad FL with Gaussian components at 605, 655 and $695 \mathrm{~nm}$ along with a sharp feature at $576 \mathrm{~nm}$. The sharp peak is band-edge transition of $\mathrm{NV}^{\circ}$ centers. ${ }^{52}$ ND0-IRR sample shows broad emission peaks of the sidebands of $\mathrm{NV}^{\circ}$ and $\mathrm{NV}^{-}$but zero phonon line (ZPL) of these color centers are unresolvable. The emission intensity of ND0IRR for side bands and ZPL is weaker as compared to ND35R8HCLIRR and ND35-OXDHCLIRR samples. It is due to the exciton trapping at outer $\mathrm{sp}^{2}$ surface layers of ND0-IRR which are diminished after oxidation. ND35-R8HCLIRR shows two prominent sharp peaks at $575 \mathrm{~nm}\left(\mathrm{NV}^{\circ}\right)$ and $638 \mathrm{~nm}\left(\mathrm{NV}^{-}\right)$ sitting over broad intense FL in the range 525-750 $\mathrm{nm}$. Broad emission can be resolved into four Gaussian components at 605 $\mathrm{nm}, 658 \mathrm{~nm}, 660 \mathrm{~nm}$ and $698 \mathrm{~nm}$ respectively. These components are the vibronic side band emission owing to the 


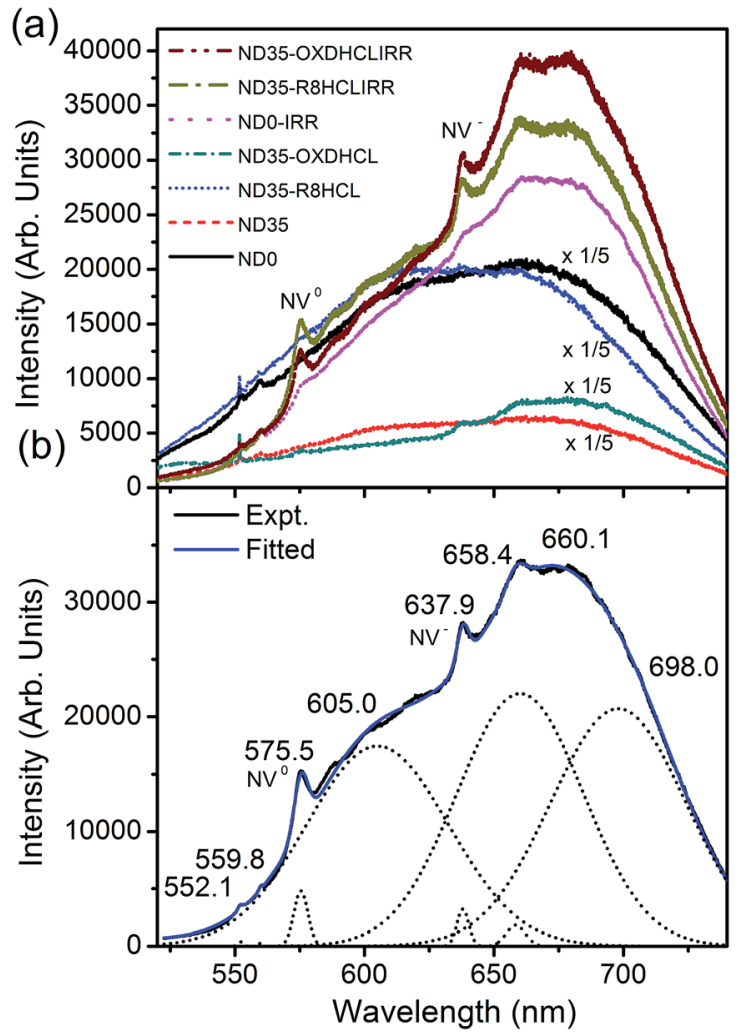

Fig. 6 (a) Shows fluorescence spectra of NDs before irradiation (ND0, ND35, ND35-R8HCL, ND35-OXDHCL) and after irradiation (ND0-IRR, ND35-R8HCLIRR, ND35-OXDHCLIRR). The intensity of curves for NDs before irradiation (ND0, ND35, ND35-R8HCL, ND35-OXDHCL) have been scaled up by a factor of 5 for clear visualization. (b) FL spectra were fitted using Gaussian components, typically shown for ND35R8HCLIRR. It shows characteristic emission peak at 575.5 and 637.9 $\mathrm{nm}$ of $\mathrm{NV}^{\circ}$ and $\mathrm{NV}^{-}$defect centers along with broad vibronic side band emission extended up to $750 \mathrm{~nm}$.

electron-phonon interaction at room temperature. Similarly, ND35-OXDHCLIRR shows sharp emission peaks at $575.1 \mathrm{~nm}$ $\left(\mathrm{NV}^{\circ}\right)$ and $637.8 \mathrm{~nm}\left(\mathrm{NV}^{-}\right)$along with broad emission peaks. The broad emission peak of ND35-OXDHCLIRR can also be resolved into four Gaussian components centered at 600.8, 658.6, 675.6 and $715.8 \mathrm{~nm}$ (vibronic side bands). Fitted spectral parameters show that the emission intensity due to $\mathrm{NV}^{-}$centers is much stronger than the emission intensity due to $\mathrm{NV}^{\circ}$ centers in ND35-OXDHCLIRR. This is in contrast to the ND35R8HCLIRR where $\mathrm{NV}^{\circ}$ centers shows the more intense ZPL intensity than $\mathrm{NV}^{-}$. The ND35-OXDHCLIRR NDs shows stronger emission in the red region than the ND35-R8HCLIRR NDs.

\section{Conclusions}

We have shown that the fluorescence from NV center engineered NDs depends upon the oxidation methods. We observed that the diamond particles can be de-agglomerated into smaller particles upon ball milling, while crystallite size does not change. Sample purified through acid reflux shows smaller size
NDs than the air oxidized ones. Acid refluxed NDs were more water dispersible than the air oxidized samples. Acid refluxed NDs were primarily rectangular in shapes while air oxidized NDs contained equal mixture of rectangular and irregular shaped particles. Purification by air oxidation was observed to be more efficient for $\mathrm{sp}^{2}$ carbon removal from Raman spectroscopy and contained less defects. While on the outer surface, particles contained higher density of $\mathrm{sp}^{2}$ carbon in case of air oxidized samples as revealed through XPS. Sample purified through air oxidation shows relatively intense fluorescence than purified through acid reflux. Since for bio-imaging applications water dispersible NDs with defined shape are desirable with sufficient FL intensity, purification by acid reflux should be preferred for such uses. While for nano-photonic applications, where the irregularity of diamond particle shape is not a real concern; purification by air oxidation is recommended which gives intense FL intensity. Thus depending upon the end use of NDs, whether it is bio-photonics or nano-photonics, we need to follow purification by acid reflux and air oxidation respectively.

\section{Acknowledgements}

Dilip K. Singh thanks DST, Govt. of India for financial support through Inspire faculty award (IFA-13 PH-65).

\section{References}

1 P. Pantazis, J. Maloney, D. Wu and S. E. Fraser, Proc. Natl. Acad. Sci. U. S. A., 2010, 107, 14535-14540.

2 E. Rittweger, K. Y. Han, S. E. Irvine, C. Eggeling and S. W. Hell, Nat. Photonics, 2009, 3, 144-147.

3 K. I. Willig, B. Harke, R. Medda and S. W. Hell, Nat. Methods, 2007, 4, 915-918.

4 M. J. Rust, M. Bates and X. Zhuang, Nat. Methods, 2006, 3, 793-796.

5 P. Annibale, S. Vanni, M. Scarselli, U. Rothlisberger and A. Radenovic, PLoS One, 2011, 6, e22678.

6 Y. Zhu, J. Li, W. Li, Y. Zhang, X. Yang, N. Y. Sun, Y. Zhao, C. Fan and Q. Huang, Theranostics, 2012, 2, 302.

7 S. Yu, M. Kang, H. Chang, K. Chen and Y. Yu, J. Am. Chem. Soc., 2005, 127, 17604-17605.

8 A. gruber, A. Dräbenstedt, C. Tietz, L. fleury, J. wrachtrup and C. von borczyskowski, Science, 1997, 276, 2012-2014.

9 F. Jelezko, C. Tietz, A. Gruber, I. Popa, A. Nizovtsev, S. Kilin and J. Wrachtrup, Single Mol., 2001, 2, 255-260.

10 F. Jelezko and J. Wrachtrup, Phys. Status Solidi A, 2006, 203, 3207.

11 Y. Chen, H. Shu, Y. Kuo, Y. Tzeng and H. Chang, Diamond Relat. Mater., 2011, 20, 803-807.

12 J. Boudou, P. Curmi, F. Jelezko, J. Wrachtrup, P. Aubert, M. Sennour, G. Balasubramanian, R. Reuter, A. Thorel and E. Gaffet, Nanotechnology, 2009, 20, 235602.

13 V. N. Mochalin, O. Shenderova, D. Ho and Y. Gogotsi, Nat. Nanotechnol., 2012, 7, 11-23.

14 V. Y. Dolmatov, Russ. Chem. Rev., 2001, 70, 607.

15 G. Yang, J. Wang and Q. Liu, J. Phys.: Condens. Matter, 1998, 10, 7923. 
16 A. Stacey, I. Aharonovich, S. Prawer and J. E. Butler, Diamond Relat. Mater., 2009, 18, 51-55.

17 C. Kittel, Introduction to Solid State Physics, 8th edition, 2012.

18 S. Osswald, G. Yushin, V. Mochalin, S. O. Kucheyev and Y. Gogotsi, J. Am. Chem. Soc., 2006, 128, 11635-11642.

19 L. L. Huang and H. Chang, Langmuir, 2004, 20, 5879-5884.

20 S. Stehlik, M. Varga, M. Ledinsky, V. Jirasek, A. Artemenko, H. Kozak, L. Ondic, V. Skakalova, G. Argentero, T. Pennycook, J. C. Meyer, A. Fejfar, A. Kromka and B. Rezek, J. Phys. Chem. C, 2015, 119, 27708-27720.

21 C. A. Schneider, W. S. Rasband and K. W Eliceiri, Nat. Methods, 2012, 9, 671-675.

22 P. K. Giri, S. Bhattacharyya, D. K. Singh, R. Kesavamoorthy, B. K. Panigrahi and K. G. M. Nair, J. Appl. Phys., 2007, 102, 093515.

23 A. Pentecost, S. Gour, V. Mochalin, I. Knoke and Y. Gogotsi, ACS Appl. Mater. Interfaces, 2010, 2, 3289-3294.

24 N. Wada and S. A. Solin, Physica B+C, 1981, 105, 353-356.

25 A. C. Ferrari and J. Robertson, Philos. Trans. R. Soc., B, 2004, 362, 2477.

26 A. Jorio, R. Saito, G. Dresselhaus and M. S. Dresselhaus, Raman spectroscopy in Graphene related systems, Wiley $\mathrm{VCH}$ 2010.

27 D. K. Singh, P. K. Iyer and P. K. Giri, J. Appl. Phys., 2012, 111, 064304.

28 Y. Kawashima and G. Katagiri, Phys. Rev. B: Condens. Matter Mater. Phys., 1995, 52, 10053.

29 A. C. Ferrari and J. Robertson, Phys. Rev. B: Condens. Matter Mater. Phys., 2001, 63, 121405.

30 A. C. Ferrari and J. Robertson, Philos. Trans. R. Soc., A, 2004, 362, 2269-2270.

31 D. K. Singh, P. K. Iyer and P. K. Giri, Diamond Relat. Mater., 2010, 19, 1281.

32 F. Y. Xie, W. G. Xie, J. Chen, X. Liu, D. Y. Lu and W. H. Zhang, J. Vac. Sci. Technol., B, 2008, 26, 102-105.

33 M. J. Webb, P. Palmgren, P. Pal, O. Karis and H. Grennberg, Carbon, 2011, 49, 3242-3249.

34 I. Kusunoki, M. Sakai, Y. Igari, S. Ishidzuka, T. Takami, T. Takaoka, M. nishiani- Gamo and T. ando, Surf. Sci., 2001, 492, 315-328.
35 Y. Fan, A. G. Fizgerald, P. John, C. E. Troupe and J. I. B. Wilson, Surf. Interface Anal., 2002, 34, 703-707.

36 H. Estrade-Szwarckopf, Carbon, 2004, 42, 1713-1721.

37 G. Cunningham, A. M. Panich, A. I. Shames, I. Petrov and O. shenderova, Diamond Relat. Mater., 2008, 17, 650-654.

38 B. Bodenant, F. Fages and M. Delville, J. Am. Chem. Soc., 1998, 120, 7511-7519.

39 I. Aharonovich, S. Castelletto, D. A. Simpson, C. Su, A. D. Greentree and S. Prawer, Rep. Prog. Phys., 2011, 74, 076501.

40 D. K. Singh, P. K. Iyer and P. K. Giri, J. Appl. Phys., 2012, 111, 064304.

41 Y. Kawashima and G. Katagiri, Phys. Rev. B: Condens. Matter Mater. Phys., 1995, 52, 10053.

42 A. C. Ferrari and J. Robertson, Phys. Rev. B: Condens. Matter Mater. Phys., 2001, 63, 121405.

43 A. C. Ferrari and J. Robertson, Philos. Trans. R. Soc. London, Ser. A, 2004, 362, 2269-2270.

44 D. K. Singh, P. K. Iyer and P. K. Giri, Diamond Relat. Mater., 2010, 19, 1281.

45 F. Y. Xie, W. G. Xie, J. Chen, X. Liu, D. Y. Lu and W. H. Zhang, J. Vac. Sci. Technol., B: Microelectron. Nanometer Struct.Process., Meas., Phenom., 2008, 26, 102-105.

46 M. J. Webb, P. Palmgren, P. Pal, O. Karis and H. Grennberg, Carbon, 2011, 49, 3242-3249.

47 I. Kusunoki, M. Sakai, Y. Igari, S. Ishidzuka, T. Takami, T. Takaoka, M. Nishiani-Gamo and T. Ando, Surf. Sci., 2001, 492, 315-328.

48 Y. Fan, A. G. Fizgerald, P. John, C. E. Troupe and J. I. B. Wilson, Surf. Interface Anal., 2002, 34, 703-707.

49 H. Estrade-Szwarckopf, Carbon, 2004, 42, 1713-1721.

50 G. Cunningham, A. M. Panich, A. I. Shames, I. Petrov and

O. Shenderova, Diamond Relat. Mater., 2008, 17, 650-654.

51 B. Bodenant, F. Fages and M. Delville, J. Am. Chem. Soc., 1998, 120, 7511-7519.

52 I. Aharonovich, S. Castelletto, D. A. Simpson, C. Su, A. D. Greentree and S. Prawer, Rep. Prog. Phys., 2011, 74, 076501. 